\title{
Searching for gas-rich disks around T Tauri stars in Lupus ${ }^{\star}$
}

\author{
T. A. van Kempen ${ }^{1}$, E. F. van Dishoeck ${ }^{1}$, C. Brinch ${ }^{1}$, and M. R. Hogerheijde ${ }^{1}$ \\ Leiden Observatory, PO Box 9513, 2300 RA Leiden, The Netherlands \\ e-mail: kempen@strw.leidenuniv.nl
}

Received 9 March 2006 / Accepted 7 June 2006

ABSTRACT

\begin{abstract}
Aims. We characterize the molecular environment of classical T Tauri stars in Lupus observed with the Spitzer Space Telescope in our search for gas-rich disks toward these sources.

Methods. Submillimeter observations of ${ }^{12} \mathrm{CO},{ }^{13} \mathrm{CO}$, and $\mathrm{C}^{18} \mathrm{O}$ in the $J=3-2$ and $2-1$ lines were obtained with the James Clerk Maxwell Telescope toward a sample of $21 \mathrm{~T}$ Tauri stars with disks in the Lupus molecular clouds. Pointings at the sources and at selected off-positions are presented in order to disentangle material associated with disks from ambient cloud material.

Results. One source, IM Lup (Sz 82), was found with the double-peaked ${ }^{12} \mathrm{CO}$ and ${ }^{13} \mathrm{CO}$ profiles characteristic of a large rotating gas disk. The inclination of the disk is found to be $\sim 20^{\circ}$, with an outer radius of $400-700$ AU. For most other sources, including GQ Lup with its substellar companion, the single-dish ${ }^{12} \mathrm{CO}$ lines are dominated by extended cloud emission with a complex velocity structure. No evidence of molecular outflows is found. Due to dense circumstellar material, compact $\mathrm{C}^{18} \mathrm{O}$ emission was detected toward only two sources. Future searches for gas-rich disks in Lupus should either use interferometers or perform very deep singledish integrations in dense gas tracers to separate the cloud and disk emission.
\end{abstract}

Key words. accretion, accretion disks - radiative transfer - methods: observational - stars: pre-main sequence - radio lines: ISM stars: circumstellar matter

\section{Introduction}

A general characteristic of pre-main sequence (PMS) stars is the presence of circumstellar disks, which have been observed for a wide range of evolutionary stages associated with low-mass star formation (e.g., Lada 1987; André et al. 1993; Greaves 2005). These disks contain all the necessary ingredients for forming complex planetary systems similar to our own solar system, now also seen around other stars (e.g., Ruden 1999; Marcy \& Butler 1995). The gas-rich phase of disks is crucial in the evolution, since gas is needed to build Jovian-type planets. Also, the presence of gas affects the dynamics and evolution of the dust in the system and the migration of any planets embedded in the disk (e.g., Gorti \& Hollenbach 2004). With time, the disk will lose its gas through processes such as photoevaporation, stellar winds, formation of gas-rich planets, and accretion onto the star (e.g., Hollenbach et al. 2000; Alexander et al. 2005). However, the timescale for gas dissipation in disks is still poorly constrained observationally, and it is even unclear whether gas and dust are lost simultaneously from disks when they evolve from the massive optically-thick to the tenuous optically-thin "debris" phase.

To constrain these timescales, surveys of gas in disks in a variety of environments are needed with sensitivities that are sufficient for detecting a Jupiter mass of gas $\left(\sim 10^{-3} M_{\odot}\right)$. Surveys to date have focussed on $\mathrm{CO}$ millimeter observations, mostly of disks in the Taurus molecular cloud (e.g. Thi et al. 2001). Although CO is known to be a poor tracer of the gas mass due to the combined effects of photodissociation in the upper layers and freeze-out in the midplane (e.g., van Zadelhoff et al. 2003), it is much simpler to observe with current instrumentation

* Figure 6 is only available in electronic form at http://www . aanda.org than other tracers, including $\mathrm{H}_{2}$ (Thi et al. 2001) or atomic finestructure lines (Kamp et al. 2003). Interferometer surveys have detected gas-rich disks around a large fraction $(>60 \%)$ of classical T Tauri stars in Taurus-Auriga with ages up to a few Myr and gas masses of at least a few times $10^{-4} M_{\odot}$, depending on assumptions about the CO abundance (Koerner \& Sargent 1995; Dutrey et al. 1996, 2003). In contrast, the detection rate is only $10 \%$ for the more evolved weak-line T Tauri stars (Duvert et al. 2000). Similar statistics are found in a single-dish submillimeter survey of $\mathrm{CO}$ in a spatially more distributed sample of Herbig Ae and Vega excess stars (Dent et al. 2005) and in CO infrared surveys (Najita et al. 2003; Blake \& Boogert 2004).

Surprisingly little is known about the presence of gas-rich disks around $\mathrm{T}$ Tauri stars in other nearby star-forming regions such as Corona Australis, Lupus, and Chamaeleon. This is largely due to the absence of interferometers in the Southern sky, and even Ophiuchus has been poorly sampled with current facilities. We present here an initial search for gas-rich disks in the Lupus molecular clouds, using a single-dish telescope.

The Lupus clouds, located at around galactic coordinates $335^{\circ} \leq l \leq 341^{\circ}$ and $7^{\circ} \leq b \leq 17^{\circ}$ (Hughes et al. 1994), are among the closest star-forming regions to the Sun at $\sim 150 \mathrm{pc}$ (see reviews by Krautter \& Reipurth 1991; Comerón 2006). Mid M-type stars dominate the stellar population of Lupus, which contains no confirmed O or B-type stars. In total 69 premain sequence (PMS) objects were found by Schwartz (1977), distributed over four clouds now known as Lupus 1-4 (see also Hughes et al. 1994). In addition, some 130 new weakline $\mathrm{T}$ Tauri stars were detected in ROSAT images (Krautter et al. 1994, 1997). The most massive stars in the complex are two A-type stars, HR 5999 and HR 6000, present in the subgroup that is richest in stars and complexity, Lupus 3. A good overview of the clouds is given by the extinction map made by 
Cambrésy (1999), based on star counts. Recently a higher resolution $\left(\sim 30^{\prime \prime}\right)$ map was published by Teixeira et al. (2005).

The Lupus clouds have been studied at millimeter wavelengths both in molecular emission lines and in the continuum. ${ }^{12} \mathrm{CO}$ (Tachihara et al. 2001), ${ }^{13} \mathrm{CO}$ (Tachihara et al. 1996) and $\mathrm{C}^{18} \mathrm{O}$ (Hara et al. 1999) $J=1-0$ large-scale maps were made at 2.6 resolution with the NANTEN telescope. Higher resolution observations were done with the Swedish-ESO Submillimeter Telescope (SEST) over more limited regions with $\sim 45^{\prime \prime}$ resolution by Gahm et al. (1993) (mostly ${ }^{12} \mathrm{CO} 1-0$ in Lupus 2), Rizzo et al. (1998) $\left({ }^{12} \mathrm{CO} 1-0\right.$ in Lupus 1 and 4 filaments) and Vilas-Boas et al. (2000) $\left({ }^{13} \mathrm{CO}\right.$ and $\mathrm{C}^{18} \mathrm{O} 1-0$ in localized dark cores in Lupus 1-4). Nürnberger et al. (1997) performed a continuum survey of $32 \mathrm{~T}$ Tauri stars in Lupus at $1.3 \mathrm{~mm}$ with the SEST, probing the cold circumstellar dust.

The Lupus star-forming region appears to differ in several aspects from other low-mass star-forming clouds. In contrast to Ophiuchus and Taurus, which contain a significant fraction of deeply embedded Class 0 and Class I objects, Lupus has at most a few embedded objects (Krautter \& Reipurth 1991; Tachihara et al. 1996; Comerón 2006). This could be an indication that Lupus is more evolved than other star-forming regions. The peak in the age distribution is $3 \times 10^{6} \mathrm{yr}$ for stars in Lupus 3 and 4, calculated from the PMS evolutionary tracks of D'Antona \& Mazzitelli (1994) for a distance of 150 pc (Hughes et al. 1994). Under the same assumptions, Lupus 1 and 2 PMS stars are younger with a peak of $1 \times 10^{6} \mathrm{yr}$. These age estimates are comparable to those for other star-forming regions such as Chamaeleon (Hartigan 1993), but are about double that of the Taurus cloud (Simon et al. 1993). Thus, a study of disks in Lupus is interesting because this is precisely the age range in which significant evolution of the gas disk is expected.

A second characteristic of the Lupus clouds is the distribution of spectral types. Compared to the Taurus cloud, Lupus is dominated by lower-mass stars. The peak of the distribution in spectral type is M0, with very few stars having spectral types higher than K7. In contrast, the peak of the spectral type distribution in Taurus is at $\mathrm{K} 7$.

In this work, single-dish submillimeter observations of $\mathrm{CO}$ toward a sample of 21 classical T Tauri stars with disks are presented. They were obtained with a beam of $14^{\prime \prime}$, much smaller than that of previous data. The sources are a subset of the PMS stars found by Schwartz (1977), Krautter \& Reipurth (1991), and Hughes et al. (1994), and they have been observed with the Spitzer Space Telescope in the context of the "From Molecular Cores to Planet Forming Disks" (c2d) Legacy survey (Evans et al. 2003). The aim of the CO observations is to characterize the molecular environment of the T Tauri stars at high angular resolution and to search for gas-rich disks suitable for future follow-up interferometer observations.

\section{Observations}

The submillimeter $\mathrm{CO}$ observations were carried out in two runs in April and July 2005 at the James Clerk Maxwell Telescope $(\mathrm{JCMT})^{1}$. The ${ }^{12} \mathrm{CO} J=3-2$ and $\mathrm{C}^{18} \mathrm{O} J=3-2$ and $2-1$ transitions (depending on weather conditions) were observed with the dual-polarization $\mathrm{B} 3$ and single-polarization $\mathrm{A} 3$ receivers.

1 The JCMT is operated at the summit of Mauna Kea, Hawaii by the Joint Astronomy Center on behalf of the United Kingdom Particle Physics and Astronomy Research Council, the Netherlands Organization for Scientific Research, and the National Research Council of Canada.
The beam sizes are $14^{\prime \prime}$ and $20^{\prime \prime}$, respectively. The Digital Autocorrelator Spectrometer was used as the back-end with a bandwidth of $125 \mathrm{MHz}$, giving a spectral resolution of $98 \mathrm{kHz}$ for the A-receiver corresponding to $0.10 \mathrm{~km} \mathrm{~s}^{-1}$ at $230 \mathrm{GHz}$, and $196 \mathrm{kHz}$ for the dual-polarization B-receiver corresponding to $0.22 \mathrm{~km} \mathrm{~s}^{-1}$ at $345 \mathrm{GHz}$. The integration times were such that the rms is around $70-100 \mathrm{mK}$ in a $0.2 \mathrm{~km} \mathrm{~s}^{-1}$ velocity bin for the B-receiver and $50 \mathrm{mK}$ for the A-receiver, depending on source and observing conditions, on a $T_{\mathrm{mb}}$ scale. Pointing was checked regularly for these southern sources and found to vary within $2^{\prime \prime}$. Beam efficiencies were taken to be 0.65 for RxA3 and 0.63 for $\mathrm{RxB}^{2}$.

Frequency switching with a switch of $16.4 \mathrm{MHz}$ was adopted for ${ }^{12} \mathrm{CO}$ because no emission-free position could readily be found for many of the sources in our sample. If a signal was detected on source, additional observations were carried out at $30^{\prime \prime}$ offsets to the east and south of the source to characterize its environment and constrain the on-source contribution. If indications were found of a gas-rich disk, additional follow-up observations were done on-source in ${ }^{13} \mathrm{CO} 3-2$ using a beam switch of $180^{\prime \prime}$ in AzEl. $\mathrm{C}^{18} \mathrm{O} 3-2$ or 2-1 observations were performed for nearly all sources, also using a beam switch of $180^{\prime \prime}$. Table 1 summarizes the observed lines per source. The data were reduced with the SPECX and CLASS reduction packages.

Our observing strategy is similar to that for the single-dish observations of gas-rich disks in Taurus by Thi et al. (2001). Because the surface layers of disks are warm, the ${ }^{12} \mathrm{CO} \mathrm{3-2}$ line is well-suited to observing these regions. Also, the smaller beam size at higher frequencies is more favorable to observing disks, which have typical sizes of a few hundred AU corresponding to a few arcsec at the distance of Lupus. Typical ${ }^{12} \mathrm{CO} 3-$ 2 antenna temperatures of disks in Taurus are $0.5-1 \mathrm{~K}$, so that an rms of $<0.1 \mathrm{~K}$ needs to be reached. To distinguish gas associated with disks from that of surrounding clouds, observations on-source and at nearby off-source positions need to be taken. Gas-rich disks should show a characteristic double-peaked line profile and a stronger signal on-source than at the off-source positions, while cloud emission should produce a comparable signal at all positions. Finally, disks in Taurus have not been detected in $\mathrm{C}^{18} \mathrm{O}$ with single-dish telescopes. On the other hand, such beam-switched $\mathrm{C}^{18} \mathrm{O}$ observations have been found to be a good probe of any dense circumstellar gas or remnant envelope associated with the source. For example, Class I sources show typical $\mathrm{C}^{18} \mathrm{O}$ intensities of $0.5-2 \mathrm{~K}$ (Hogerheijde et al. 1998; Jørgensen et al. 2002).

Our sample of classical T Tauri stars in Lupus was selected from targets belonging to the InfraRed Spectrometer (IRS) sample of the c2d Legacy program on Spitzer (see Table 1). They are a subset of the T Tauri stars studied by Schwartz (1977), Krautter \& Reipurth (1991) and Hughes et al. (1994), selected to be brighter than $200 \mathrm{mJy}$ at $12 \mu \mathrm{m}$ and excluding sources contained in the Spitzer-IRS guaranteed time programs. Infrared photometry and spectroscopy was performed on all sources giving estimates of their spectral types (see Table 1). In total 21 PMS stars were observed, most of them located in Lupus 3. All sources show a clear $10 \mu \mathrm{m}$ emission feature due to warm silicates, confirming the presence of a circumstellar disk (Kessler-Silacci et al. 2006). Some of the selected stars are known binaries. Where available, results of the $1.3 \mathrm{~mm}$ continuum emission are reported (Nürnberger et al. 1997). This emission is presumably dominated by the cold dust in the disk.

\footnotetext{
2 Observed by the JCMT staff over many observations; see the JCMT website http://www. jach.hawaii . edu/JCMT/
} 
Table 1. Summary of the properties of T Tauri stars and observed lines in Lupus.

\begin{tabular}{|c|c|c|c|c|c|c|c|c|c|}
\hline Source & RA (J2000) & Dec (J2000) & Spectr. type ${ }^{a}$ & Cloud & $F_{1.3 \mathrm{~mm}}(\mathrm{mJy})^{b}$ & ${ }^{12} \mathrm{CO}$ & Offsets & $\mathrm{C}^{18} \mathrm{O}$ & Add. obs \\
\hline HT Lup ${ }^{c}$ & 154512.87 & -341730.6 & K2 & Lupus 1 & 135 & $3-2$ & E S & $3-2 / 2-1$ & \\
\hline GW Lup & 154644.68 & -343035.4 & M2-M4 & Lupus 1 & 106 & $3-2$ & E S & $3-2 / 2-1$ & \\
\hline HM Lup & 154750.63 & -352835.4 & M3 & Lupus 1 & $<45$ & $3-2$ & E S & $3-2 / 2-1$ & \\
\hline $\mathrm{Sz} 73$ & 154756.98 & -351435.1 & M0 & Lupus 1 & 26 & $3-2$ & E S & $2-1$ & \\
\hline GQ Lup & 154912.12 & -353905.0 & K7-M0 & Lupus 1 & 38 & $3-2$ & E S & $2-1$ & ${ }^{13} \mathrm{CO} 3-2$ \\
\hline IM Lup & 155609.17 & -375606.4 & M0 & Lupus 2 & 260 & $3-2$ & E S & $2-1$ & ${ }^{13} \mathrm{CO} 3-2$ \\
\hline RU Lup & 155642.31 & -374915.5 & K7-M0 & Lupus 2 & 197 & $3-2$ & E S & $3-2$ & \\
\hline $\mathrm{Sz} 84$ & 155802.52 & -373602.8 & M5.5 & Lupus 2 & $<36$ & $3-2$ & E S & $2-1$ & \\
\hline RY Lup & 155928.39 & -402151.2 & K4 & Lupus 3 & - & $3-2$ & - & $3-2$ & \\
\hline EX Lup & 160305.52 & -401824.9 & M0 & Lupus 3 & - & $3-2$ & - & $3-2$ & \\
\hline HO Lup ${ }^{c}$ & 160700.61 & -390219.4 & M1 & Lupus 3 & $<24$ & $3-2$ & E S & $3-2$ & \\
\hline $\mathrm{Sz} 96$ & 160812.64 & -390833.3 & M1.5 & Lupus 3 & $<45$ & $3-2$ & E S & $3-2$ & \\
\hline Krautter's star & 160829.70 & -390311.2 & K0 & Lupus 3 & $<30$ & $3-2$ & E S & $3-2$ & \\
\hline Sz 107 & 160841.78 & -390136.4 & M5.5 & Lupus 3 & - & $3-2$ & E S & - & \\
\hline Sz 109 & 160848.17 & -390419.0 & M5.5 & Lupus 3 & - & $3-2$ & E S & $3-2$ & \\
\hline Sz 110 & 160851.56 & -390317.5 & M2 & Lupus 3 & - & $3-2$ & E S & $3-2$ & \\
\hline Sz 111 & 160854.74 & -393717.5 & M1.5 & Lupus 3 & - & - & - & $3-2$ & \\
\hline V908 Sco & 160901.84 & $\begin{array}{lll}-39 & 05 & 12.1\end{array}$ & M4 & Lupus 3 & - & $3-2$ & E S & $3-2$ & \\
\hline Sz 117 & 160944.34 & -391330.4 & M2 & Lupus 3 & - & $3-2$ & E S & $3-2$ & \\
\hline Sz 118 & 160948.68 & -391117.2 & K6 & Lupus 3 & - & $3-2$ & E S & $3-2$ & \\
\hline Sz 123 & 161051.58 & $\begin{array}{llll}-38 & 53 & 13.7\end{array}$ & M1 & Lupus 3 & - & $3-2$ & E S & $3-2$ & \\
\hline
\end{tabular}

${ }^{a}$ Hughes et al. (1994); ${ }^{b}$ Nürnberger et al. (1997), where available; ${ }^{c}$ known binaries (Nürnberger et al. 1997).

Table 2. Observed line intensities toward T Tauri stars in Lupus.

\begin{tabular}{|c|c|c|c|c|c|c|}
\hline \multirow[t]{2}{*}{ Source } & \multicolumn{2}{|c|}{$\int T_{\mathrm{MB}} \mathrm{d} V$} & \multicolumn{4}{|c|}{ Peak $T_{\mathrm{MB}}$} \\
\hline & $\begin{array}{c}{ }^{12} \mathrm{CO} \mathrm{3}_{3-2} \\
{\left[\mathrm{~K} \mathrm{~km} \mathrm{~s}^{-1}\right]}\end{array}$ & $\begin{array}{c}{ }^{13} \mathrm{CO} \mathrm{3}^{3-2} \\
{\left[\mathrm{~K} \mathrm{~km} \mathrm{~s}^{-1}\right]}\end{array}$ & $\begin{array}{c}{ }^{12} \mathrm{CO} \\
{[\mathrm{K}]}\end{array}$ & $\begin{array}{c}\mathrm{C}^{18} \mathrm{O} 3-2^{a} \\
{[\mathrm{~K}]}\end{array}$ & $\begin{array}{c}\mathrm{C}^{18} \mathrm{O} 2-1^{a} \\
{[\mathrm{~K}]}\end{array}$ & $\begin{array}{c}{ }^{13} \mathrm{CO} 3-2 \\
{[\mathrm{~K}]}\end{array}$ \\
\hline HT Lup & 26.5 & & 15 & 1.9 & 3.5 & \\
\hline GW Lup & 9.1 & & 6.7 & $<0.16$ & $<0.08$ & \\
\hline HM Lup & 6.4 & & 5.2 & $<0.15$ & $<0.08$ & \\
\hline $\mathrm{Sz} 73$ & 15.8 & & 9.0 & & 1.0 & \\
\hline GQ Lup & 13.4 & $<0.14^{c}$ & 5.8 & & $<0.05$ & $<0.25^{c}$ \\
\hline IM Lup & 4.7 & 0.52 & 2.4 & & $<0.09$ & 0.35 \\
\hline RU Lup & - & & - & $<0.27$ & & \\
\hline Sz 84 & 0.6 & & 0.8 & & $<0.09$ & \\
\hline RY Lup & $b$ & & $<0.25$ & $<0.31$ & & \\
\hline EX Lup & $b$ & & $<0.16$ & $<0.40$ & & \\
\hline HO Lup & 1.3 & & 0.8 & $<0.17$ & & \\
\hline Sz 96 & 25.1 & & 12 & $<0.16$ & & \\
\hline Krautter's star & 17.5 & & 11.7 & $<0.25$ & & \\
\hline Sz 107 & 18.3 & & 12 & & & \\
\hline Sz 109 & 34.8 & & 26 & $<0.15$ & & \\
\hline Sz 110 & 26.1 & & 21 & $<0.25$ & & \\
\hline Sz 111 & $b$ & & $<0.2$ & $<0.30$ & & \\
\hline V908 Sco & 22.6 & & 13.7 & $<0.26$ & & \\
\hline Sz 117 & 11.4 & & 5.5 & $<0.22$ & & \\
\hline Sz 118 & 14.3 & & 7.2 & $<0.27$ & & \\
\hline Sz 123 & 2.4 & & 1.6 & $<0.20$ & & \\
\hline
\end{tabular}

${ }^{a}$ If no line was detected, a $2 \sigma_{\text {rms }}$ (see Sect. 2.1) limit in $\delta v=0.25 \mathrm{~km} \mathrm{~s}^{-1}$ is given; ${ }^{b}$ no detection. See peak value for rms; ${ }^{c}$ see Sect. 4.2 .

The distances to individual Lupus clouds have considerable uncertainty. Hughes et al. (1993) found $140 \pm 20 \mathrm{pc}$ from the spectroscopic parallax of field stars around the clouds. However, observations of reddening and interstellar absorption lines toward individual stars by, e.g., Knude \& Høg (1998) and Crawford (2000) find distances ranging from 100 to $200 \mathrm{pc}$. A good discussion can be found in Comerón (2006). The depth of the Lupus clouds may be non-neglible and can be as large as $50 \mathrm{pc}$. Also, it has been suggested that not all clouds of Lupus are related; in particular Lupus 2 could be located as far away as 360 pc (Knude \& Nielsen 2001). However, this seems unlikely, given the narrow ranges in mean velocity of the clouds (see e.g., Tachihara et al. 1996; Vilas-Boas et al. 2000; and this work Sect. 3). The distance used here for all sources is that most commonly adopted, $150 \mathrm{pc}$.

\section{Results}

The observed ${ }^{12} \mathrm{CO} 3-2$ line intensities are presented in Table 2, together with the peak intensities. Typical examples of spectra 

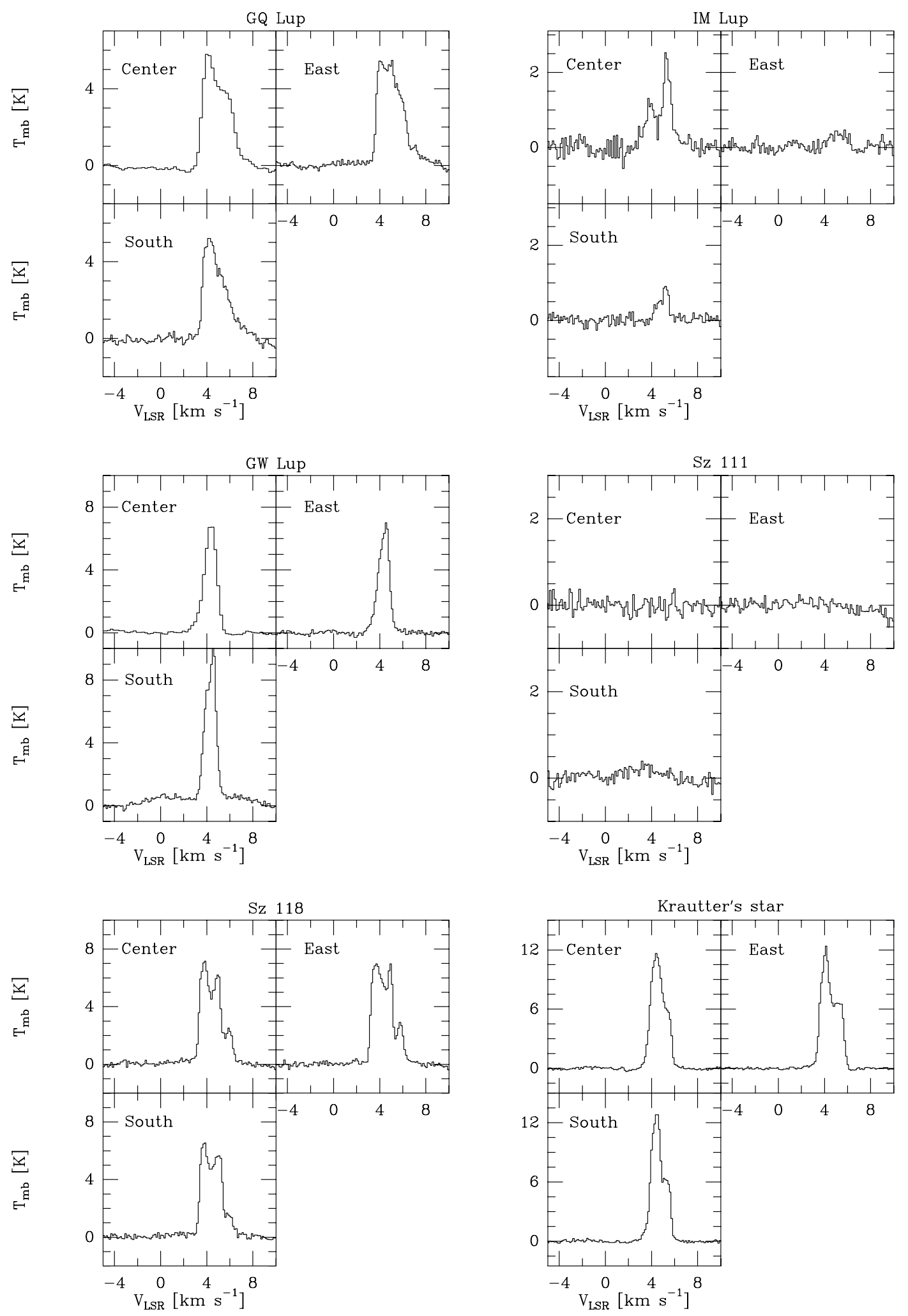

Fig. 1. ${ }^{12} \mathrm{CO} 3-2$ lines of selected sources in Lupus, with off-positions $30^{\prime \prime}$ south and east of source.

that illustrate the range of observed profiles, both on-source as well as at off-source positions, are shown in Fig. 1. The remainder of the spectra can be found in the on-line material.

Only a single source is found in our sample with a clear signal of gas associated with the source itself, namely IM Lup. A classic double-peaked disk profile is found toward the source position, which is largely absent at the $30^{\prime \prime}$ east and south positions offset from the source (see Fig. 1). The profile, with a maximum peak of $2.5 \mathrm{~K}$, is significantly broader than the one associated with quiescent gas. This source is discussed further in Sect. 4.1.

None of the other sources show indications of gas-rich disks surrounding the central T Tauri star. Three sources, RY Lup, EX Lup and Sz 111, appear to be located outside any cloud material and have no detectable disk emission at our sensitivity limit. 


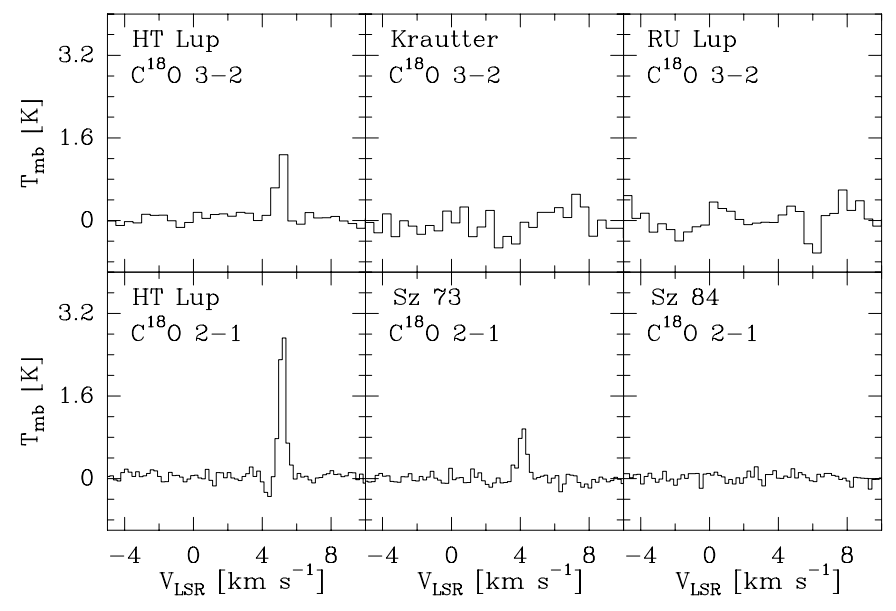

Fig. 2. $\mathrm{C}^{18} \mathrm{O}$ lines toward selected sources in Lupus.

Using the formula in Thi et al. (2001) with the observed limits on ${ }^{12} \mathrm{CO}$ emission, upper limits of the gas masses of $1 \times 10^{-6} M_{\odot}$ are obtained for these disks with a $\mathrm{CO} / \mathrm{H}_{2}$ ratio of $10^{-4}$. For a more realistic overall $\mathrm{CO} / \mathrm{H}_{2}$ disk abundance of $10^{-6}$, the limits would increase to $1 \times 10^{-4} M_{\odot}$ (see also Sect. 4).

Most sources have a strong ${ }^{12} \mathrm{CO}$ line on source, but these signals are also detected at the off-positions with comparable strengths (see Fig. 1). These lines must originate in the largescale molecular cloud material in the line of sight of the source. The complex profiles seen for many sources indicate a complex structure of different molecular clouds moving at different velocities, either in the fore- or background. For example, the spectra toward $\mathrm{Sz} 118$ (see Fig. 1) show three peaks with different intensities at all positions with widths of $\sim 0.8 \mathrm{~km} \mathrm{~s}^{-1}$ and velocities that differ by $1-1.5 \mathrm{~km} \mathrm{~s}^{-1}$. The $\mathrm{C}^{18} \mathrm{O}$ molecule is not detected at all for this source. Comparison with the large scale maps shows that observations of ${ }^{12} \mathrm{CO} 3-2$ with peak temperatures of 2-20 Kelvin are consistent with the lower-resolution ${ }^{12}$ CO surveys (Gahm et al. 1993; Tachihara et al. 1996).

The ${ }^{12} \mathrm{CO}$ line widths of $\sim 0.8 \mathrm{~km} \mathrm{~s}^{-1}$ are comparable to those found in Vilas-Boas et al. (2000) and Tachihara et al. (2001), taking multiple clouds at different velocities into account. No evidence of line wings at $\geq 2 \mathrm{~km} \mathrm{~s}^{-1}$ from line center is found at the level $T_{\mathrm{MB}}<0.15 \mathrm{~K}(2 \sigma)$, providing limits on any smallscale molecular outflows associated with these T Tauri stars. For RU Lup, this is consistent with Gahm et al. (1993), who reported no outflows from CO 1-0 data in a larger beam.

Although no direct correlation can be inferred due to the confusion of cloud emission around most sources, it is interesting to note that the largest $1.3 \mathrm{~mm}$ flux found by Nürnberger et al. (1997) is associated with IM Lup. Thus it also contains the most surrounding dust, in addition to being the only source with $\mathrm{CO}$ disk emission.

The results for the $\mathrm{C}^{18} \mathrm{O}$ and ${ }^{13} \mathrm{CO}$ line intensities are included in Table 2 . The ${ }^{13} \mathrm{CO} 3-2$ molecule was observed for only two objects: IM Lup and GQ Lup. A more detailed description for these two objects can be found in Sect. 4. Figure 2 shows some examples of observed $\mathrm{C}^{18} \mathrm{O}$ lines.

Only two sources in our sample show a $\mathrm{C}^{18} \mathrm{O}$ line above the noise level, HT Lup and Sz 73, and HT Lup is the only source toward which both $\mathrm{C}^{18} \mathrm{O} 2-1$ and 3-2 lines have been detected in our survey. Because the data were taken in $180^{\prime \prime}$ beam-switched mode, the bulk of the emission is likely to be directly associated with the source. This could point towards a small circumstellar envelope. The 2-1/3-2 line ratio of
$>1$ indicates either very cold gas $(<15 \mathrm{~K})$ or densities less than $10^{5} \mathrm{~cm}^{-3}$ for $T \approx 20 \mathrm{~K}$. The inferred $\mathrm{C}^{18} \mathrm{O}$ column density for these conditions is $\sim 10^{15} \mathrm{~cm}^{-2}$, corresponding to a CO column density of $5 \times 10^{17} \mathrm{~cm}^{-2}$ for ${ }^{16} \mathrm{O} /{ }^{18} \mathrm{O}=540$ (Wilson \& Rood 1994) and an $\mathrm{H}_{2}$ column density of $6 \times 10^{21} \mathrm{~cm}^{-2}$ for a dark cloud $\mathrm{C}^{18} \mathrm{O}$ abundance of $1.7 \times 10^{-7}$ (Frerking et al. 1982). Thus, the extinction directly associated with the source is $A_{V} \approx 6 \mathrm{mag}$ using $N_{\mathrm{H}} / A_{\mathrm{V}}=1.8 \times 10^{21} \mathrm{~cm}^{-2} \mathrm{mag}^{-1}$ (Rachford et al. 2002).

As mentioned before, our observations are not sensitive to extended $\mathrm{C}^{18} \mathrm{O}$ emission because of beam-switching by $3^{\prime}$. The lack of any features at the $2 \sigma$ level of $<0.1 \mathrm{~K}$ in $\mathrm{C}^{18} \mathrm{O} 2-1$ and $<0.3 \mathrm{~K}$ in 3-2 implies that either any large scale cloud emission must be very smooth on a $3^{\prime}$ scale or that there is no $\mathrm{C}^{18} \mathrm{O}$ at this limit. Vilas-Boas et al. (2000) and Hara et al. (1999) find $\mathrm{C}^{18} \mathrm{O} 1-0$ antenna temperatures of 0.2 to $1.5 \mathrm{~K}$ for the densest cores. For typical dark cloud conditions, the $2-1$ line should be comparable in strength, whereas the 3-2 line should be factors of 2-4 weaker, so that these lines would have been detectable at the positions of strongest $1-0$ emission. However, most of our sources are located at the edge or outside the $\mathrm{C}^{18} \mathrm{O}$ contours of Hara et al. (1999), suggesting that $\mathrm{C}^{18} \mathrm{O}$ is indeed largely absent. The lack of $\mathrm{C}^{18} \mathrm{O} 2-1$ emission at the level of $0.08 \mathrm{~K}(2 \sigma)$ indicates a $\mathrm{C}^{18} \mathrm{O}$ column density of less than $5 \times 10^{13} \mathrm{~cm}^{-2}$, or $\mathrm{H}_{2}$ less than $3 \times 10^{20} \mathrm{~cm}^{-2}$ using the dark cloud abundance. In this diffuse cloud regime, however, the $\mathrm{C}^{18} \mathrm{O}$ abundance may be significantly lower than the standard dark cloud value (van Dishoeck \& Black 1988), increasing the $\mathrm{H}_{2}$ column density.

\section{Individual objects}

Several objects showed interesting enough spectra to probe deeper. IMLup and GQLup are presented here with a more indepth analysis.

IM Lup is the only T Tauri star in our sample with a clear detection of ${ }^{12} \mathrm{CO}$ emission associated with the source. A doublepeaked profile with peak separations of about $2 \mathrm{~km} \mathrm{~s}^{-1}$ is seen in both ${ }^{12} \mathrm{CO}$ and ${ }^{13} \mathrm{CO}$, consistent with a rotating gas disk (see Fig. 3). In addition, the lack of $\mathrm{C}^{18} \mathrm{O}$ emission over $0.09 \mathrm{~K}$ in a $0.25 \mathrm{~km} \mathrm{~s}^{-1}$ bin implies the absence of a significant contribution of a dense circumstellar envelope. The existence of a large disk around IM Lup has recently been shown in scattered light images at visible and near-infrared wavelengths with the Hubble Space Telescope (Padgett et al., priv. comm; Schneider et al., priv. comm.).

The ${ }^{12} \mathrm{CO}$ peak intensity for IM Lup is comparable to that of the brighter sources with gas-rich disks in Taurus observed by Thi et al. (2001). The much weaker ambient cloud material seen at the south off-position (see Fig. 1) modestly affects the disk profile, however, especially the red peak at $V_{\mathrm{LSR}}=5 \mathrm{~km} \mathrm{~s}^{-1}$. The blue peak at $V_{\mathrm{LSR}}=3 \mathrm{~km} \mathrm{~s}^{-1}$ is not contaminated so is taken as the actual disk signal for both peaks. The ${ }^{13} \mathrm{CO}$ peaks of $0.2 \mathrm{~K}$ are comparable to those for Taurus disks and do not seem to be affected by ambient cloud material.

The observed ${ }^{12} \mathrm{CO} /{ }^{13} \mathrm{CO}$ integrated intensity ratio of $6.0 \pm 1.5$ indicates ${ }^{12} \mathrm{CO}$ and ${ }^{13} \mathrm{CO}$ optical depths of 2.3 and 0.15 , respectively, assuming that the excitation temperatures are both the same for both isotopes and constant throughout the disk. The uncertainties are dominated by calibration errors. If the peak temperatures of the blue wing are used $\left(1.5\right.$ for ${ }^{12} \mathrm{CO}$ and $0.4 \mathrm{~K}$ for ${ }^{13} \mathrm{CO}$, respectively), optical depths of 3 and 0.3 are found. 


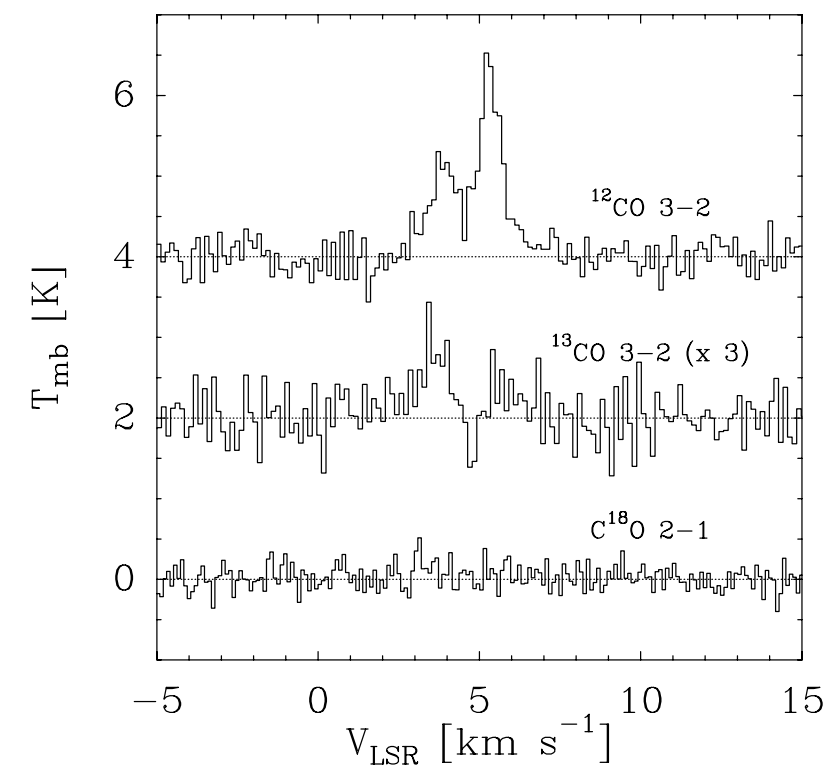

Fig. 3. ${ }^{12} \mathrm{CO} 3-2,{ }^{13} \mathrm{CO} 3-2$, and $\mathrm{C}^{18} \mathrm{O} 2-1$ spectra observed toward IM Lup (Sz 82) shifted by $+4,+2$, and $0 \mathrm{~K}$, respectively.

Thi et al. (2001) give several methods for deriving disk masses from different observations. Assuming that most of the ${ }^{13} \mathrm{CO} 3-2$ emission is optically thin and originates in the cold gas of the outer disk with a single excitation temperature, the total gas mass is given by

$$
\begin{aligned}
M_{\mathrm{gas}}=3 & \times 10^{-6}\left(\frac{\left[{ }^{12} \mathrm{CO}\right] /\left[{ }^{13} \mathrm{CO}\right]}{60}\right)\left(\frac{\mathrm{H}_{2} /{ }^{12} \mathrm{CO}}{10^{4}}\right) \\
& \times\left(\frac{T_{\mathrm{ex}}+0.89}{\mathrm{e}^{-16.02 / T_{\mathrm{ex}}}}\right)\left(\frac{\tau}{1-\mathrm{e}^{-\tau}}\right)\left(\frac{d}{100 \mathrm{pc}}\right)^{2} \int T_{\mathrm{mb}} \mathrm{d} V
\end{aligned}
$$

The $\mathrm{H}_{2} / \mathrm{CO}$ and ${ }^{12} \mathrm{CO} /{ }^{13} \mathrm{CO}$ ratios have been set to $10^{4}$ and 60 , following standard conversion factors. This results in a derived gas mass of $2 \times 10^{-4} M_{\odot}$ for IM Lup at $d=150 \mathrm{pc}$, using $T_{\mathrm{ex}} \simeq 30 \mathrm{~K}$. For comparison, the disk mass found from the $1.3 \mathrm{~mm}$ continuum flux (Nürnberger et al. 1997) is $6 \times 10^{-2} M_{\odot}$, assuming a gas/dust ratio of $100, T_{\text {dust }}=30 \mathrm{~K}$ and a dust opacity coefficient $\kappa$ of $0.01 \mathrm{~cm}^{2} \mathrm{~g}^{-1}$. The factor of 300 between these two numbers is comparable to that found for other disks (e.g., Dutrey et al. 1996; Mannings \& Sargent 2000; Thi et al. 2001) and is commonly explained by the fact that $\mathrm{CO}$ is not a good tracer of the gas mass due to the combined effects of photodissociation by stellar and interstellar UV radiation and of the freeze-out of $\mathrm{CO}$ onto the grains in cold regions of the disk (van Zadelhoff et al. 2001; Aikawa et al. 2002).

The ${ }^{12} \mathrm{CO}$ profile can be used to obtain estimates of two other important disk parameters, the disk size and inclination. Assuming optically thick ${ }^{12} \mathrm{CO} 3-2$ emission and following the method described in Dutrey et al. (1997):

$$
\int T_{\mathrm{mb}} \mathrm{d} v=T_{\mathrm{ex}}(\rho \delta v)\left(\frac{\pi\left(R_{\mathrm{out}}^{2}-R_{\mathrm{in}}^{2}\right)}{d^{2}} \cos i\right) \Omega_{a}^{-1}
$$

where $R_{\text {in }}$ and $R_{\text {out }}$ are the inner and outer radii, $\delta v$ the turbulent velocity, and $\rho$ a geometrical factor. Also, $\Omega_{a}^{-1}$ is the telescope beam on the sky and $i$ the inclination, with 0 degrees being faceon with respect to the line of sight. For IM Lup, $R_{\text {in }}=0 \mathrm{AU}$, a mean disk excitation temperature $T_{\mathrm{ex}}$ of 25,40 , and $50 \mathrm{~K}$ and $(\rho \delta v)$ of $0.3 \mathrm{~km} \mathrm{~s}^{-1}$ are chosen.

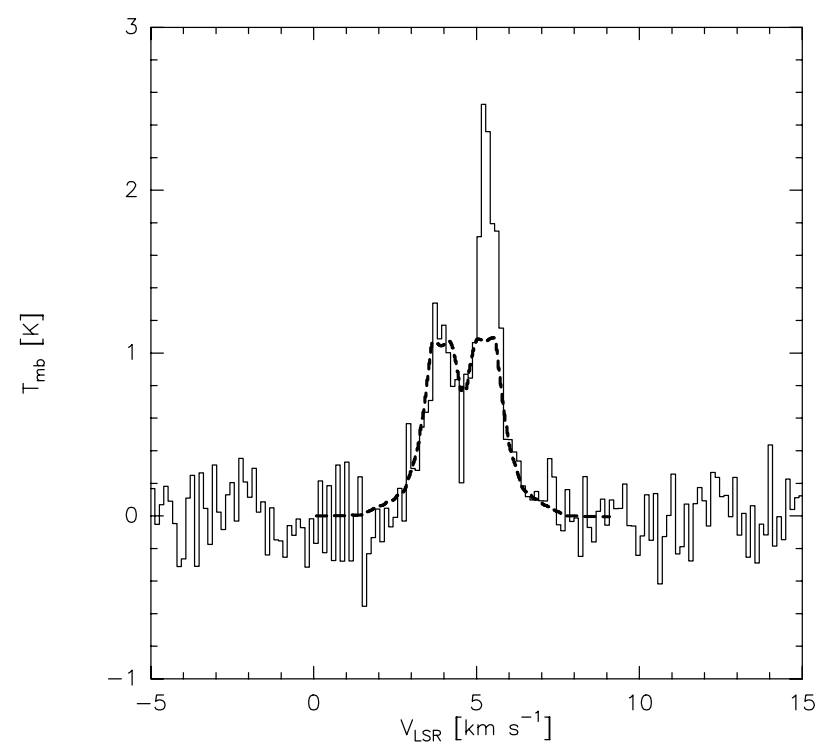

Fig. 4. Comparison of the observed ${ }^{12} \mathrm{CO} J=3-2$ line toward IM Lup with the best-model fit with a disk of $i=20^{\circ}, R=600 \mathrm{AU}$.

The disk sizes calculated from Eq. (2) are given in $R_{\text {out }} \sqrt{\cos i}$. Scattered light images indicate that the inclination is on the order of $10^{\circ}$ to $30^{\circ}$, so that $\cos i \approx 1$ is a reasonable approximation. The disk sizes found with $T_{\mathrm{ex}}=25$ and $T_{\mathrm{ex}}=40 \mathrm{~K}$ are 730 and $580 \mathrm{AU}$, respectively. Such numbers are comparable to the observed radius of the dust disk in scattered light, about $4.3^{\prime \prime}$ or $600 \mathrm{AU}$ (Padgett et al., priv. comm.; Schneider et al., priv. comm.).

To better understand the observed line profile of ${ }^{12} \mathrm{CO}$, a simple disk model has been used, together with a ray-tracing program (Hogerheijde \& van der Tak 2000) to compute the line profile. Such models are better-suited to constraining parameters using an $\chi^{2}$ method with the observed spectrum. The populations of the ${ }^{12} \mathrm{CO}$ rotational levels are assumed to be in LTE throughout the entire disk. The surface density of the disk is defined as $\Sigma(r)=\Sigma_{0}(r / 1 \mathrm{AU})^{-1.5}$ with a typical value of $4.57 \times 10^{26} \mathrm{~cm}^{-2}$ at $1 \mathrm{AU}$. The disk is assumed to be isothermal in the vertical direction. The abundance of $\mathrm{CO}$ is set to be $5 \times 10^{-5}$ with respect to $\mathrm{H}_{2}$, except in areas with temperatures below $30 \mathrm{~K}$ where $\mathrm{CO}$ is frozen out onto the grains. There, abundances are taken to be $5 \times 10^{-10}$. It is assumed that the gas is in Keplerian orbits around a $0.5 M_{\odot}$ star. The inner radius is set at $0.1 \mathrm{AU}$. The radial temperature profile is only constrained by a radial exponent of -0.5 and the luminosity. The turbulent velocity is taken to be $0.1 \mathrm{~km} \mathrm{~s}^{-1}$, following Thi et al. (2001) and Qi et al. (2004).

The parameters to be fitted are the inclination, the outer radius of the disk, and the temperature at the inner radius. The bestfit is presented in Fig. 4. It has a best fit outer radius between 400 and $700 \mathrm{AU}$, consistent with the result of $600 \mathrm{AU}$ found above. The line profile is not very sensitive to the outer radius or the $\mathrm{CO}$ abundance in the outer areas because temperatures are too low to have a significant impact on the 3-2 emission. The observed line is quite sensitive to the inclination of the disk, which is constrained to $20^{\circ} \pm 5^{\circ}$. The temperature at the inner radius is taken to be $1200 \mathrm{~K}$, but model results are not sensitive to the precise choice of temperature.

The classical T Tauri star GQ Lup has recently attracted attention because adaptive optics imaging has revealed a substellar companion, perhaps a massive Jupiter-like planet, with a separation of $\sim 100 \mathrm{AU}$ (Neuhäuser et al. 2005). As for all objects in our 


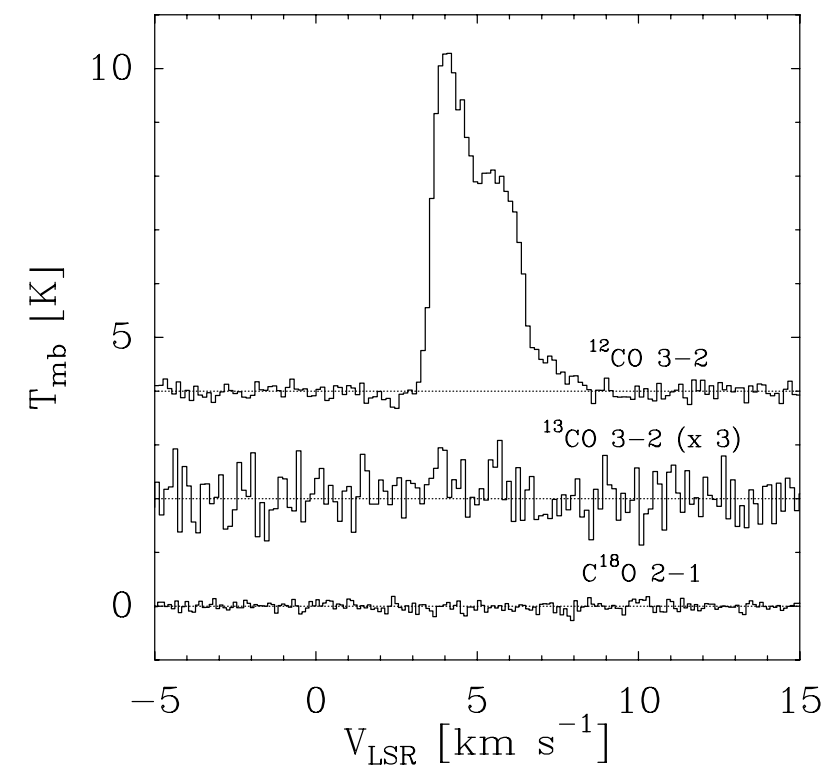

Fig. 5. ${ }^{12} \mathrm{CO} 3-2,{ }^{13} \mathrm{CO} 3-2$, and $\mathrm{C}^{18} \mathrm{O} 2-1$ lines toward GQ Lup, shifted by $+4,+2$, and $0 \mathrm{~K}$, respectively.

sample, the GQ Lup spectrum shows an infrared excess and a silicate emission feature at $10 \mu \mathrm{m}$, indicating the presence of a dust disk. This object is potentially interesting for our understanding of the influence of a companion on the disk structure and evolution. High signal-to-noise observations were taken toward this object (Fig. 5), but, like most other sources in our sample, only diffuse interstellar cloud material is detected in ${ }^{12} \mathrm{CO}$, as proven in the observations at off-positions. The ${ }^{13} \mathrm{CO}$ spectrum shows a hint of two peaks centered around $V_{\mathrm{LSR}}=5 \mathrm{~km} \mathrm{~s}^{-1}$ of equal height at $T_{\mathrm{mb}}=0.25 \mathrm{~K}$, but deeper integrations are needed to confirm this. Also, $\mathrm{C}^{18} \mathrm{O} 2-1$ was not detected at all down to $5 \mathrm{mK}(2 \sigma)$ in a $0.25 \mathrm{~km} \mathrm{~s}^{-1}$ bin. This constrains the maximum column density of $\mathrm{C}^{18} \mathrm{O}$ directly associated with the source to $\sim 2 \times 10^{12} \mathrm{~cm}^{-2}$. An unresolved disk would then be limited to a gas mass of $<2.3 \times 10^{-5} M_{\odot}$ for $\mathrm{C}^{18} \mathrm{O} / \mathrm{H}_{2}=1.67 \times 10^{-7}$ or $<2.3 \times 10^{-3} M_{\odot}$ for a lower ratio of $\mathrm{C}^{18} \mathrm{O} / \mathrm{H}_{2}=1.67 \times 10^{-9}$. The mass derived from the $1.3 \mathrm{~mm}$ flux of $38 \mathrm{mJy}$ is $2.1 \times 10^{-3}$, assuming a gas/dust ratio of $100, T_{\text {dust }}=30 \mathrm{~K}$ and a dust opacity $\kappa$ of $0.01 \mathrm{~cm}^{2} \mathrm{~g}^{-1}$ (Nürnberger et al. 1997).

\section{Concluding remarks}

The main conclusions of this work are as follows:

- A ${ }^{12} \mathrm{CO} J=3-2$ survey of 21 classical T Tauri stars with dust disks in Lupus taken in frequency-switched mode has revealed mostly extended diffuse cloud material with a few magnitudes of extinction and a complex velocity structure.

$-\mathrm{A} \mathrm{C}^{18} \mathrm{O} J=3-2$ and 2-1 survey of the same 21 sources taken in beam-switched mode shows dense compact circumstellar material associated with only 2 sources, HT Lup and $\mathrm{Sz} 73$.

- Only a single source, IM Lup (Sz 82), has been found with clear evidence of a gas-rich disk. Modeling of the line intensity and line profile indicates a large disk with radius $\sim 600 \mathrm{AU}$ and inclination $20^{\circ}$. This source will be a prime target for future interferometer observations in the southern sky, especially with the Atacama Large Millimeter Array.

That we do not detect gas disks in Lupus in the current survey does not imply that the gas has been dissipated from these disks; rather, it shows the limitations of single-dish CO line observations for revealing them. Future searches need to either (i) focus on high density single-dish tracers, such as $\mathrm{HCO}^{+} J=4-3$ or HCN $J=4-3$ to distinguish the warm, dense gas in the disk from the cold, diffuse cloud material; or (ii) use newly commisioned interferometers, such as the SubMillimeter Array (SMA). From comparison with other disks in Taurus (Thi et al. 2004; Greaves 2004), these lines are expected to have peak intensities of at most $0.1 \mathrm{~K}$ in a single-dish $15^{\prime \prime}$ beam, and it will be very difficult to detect. Increased sensitivity to compact emission is only possible with interferometer studies. Interferometric surveys will therefore be essential in determining the fraction of gas-rich disks in Lupus and thus the timescale for gas dissipation in this star-forming region compared with other regions such as Taurus.

Acknowledgements. The authors are grateful to Remo Tilanus for carrying out part of the observations and to Karl Stapelfeldt, Debbie Padgett, Glenn Schneider, and Jean-Charles Augereau for communicating information about the HST images of IM Lup before publication. T.v.K. and astrochemistry at Leiden Observatory are supported by a Spinoza grant and by grant 614.041.004 from The Netherlands Organization for Scientific Research (NWO).

\section{References}

Aikawa, Y., van Zadelhoff, G. J., van Dishoeck, E. F., \& Herbst, E. 2002, A\&A, 386,622

Alexander, R. D., Clarke, C. J., \& Pringle, J. E. 2005, MNRAS, 358, 283

André, P., Ward-Thompson, D., \& Barsony, M. 1993, ApJ, 406, 122

Blake, G. A., \& Boogert, A. C. A. 2004, ApJ, 606, L73

Cambrésy, L. 1999, A\&A, 345, 965

Comerón, F. 2006, in Handbook of southern starforming regions, ed. B. Reipurth (ESO press), in press

Crawford, I. A. 2000, MNRAS, 317, 996

D’Antona, F., \& Mazzitelli, I. 1994, ApJS, 90, 467

Dent, W. R. F. Greaves, J. S., \& Coulson, I. M. 2005, MNRAS, 359, 663

Dutrey, A., Guilloteau, S., Duvert, G., et al. 1996, A\&A, 309, 493

Dutrey, A., Guilloteau, S., \& Guelin, M. 1997, A\&A, 317, L55

Dutrey, A., Guilloteau, S., \& Simon, M. 2003, A\&A, 402, 1003

Duvert, G., Guilloteau, S., Ménard, F., Simon, M., \& Dutrey, A. 2000, A\&A, 355,165

Evans, N. J., Allen, L. E., Blake, G. A., et al. 2003, PASP, 115, 965

Frerking, M. A., Langer, W. D., \& Wilson, R. W. 1982, ApJ, 262, 590

Gahm, G. F., Johansson, L. E. B., \& Liseau, R. 1993, A\&A, 274, 415

Gorti, U., \& Hollenbach, D. 2004, ApJ, 613, 424

Greaves, J. S. 2004, MNRAS, 351, L99

Greaves, J. S. 2005, Science, 307, 68

Hara, A., Tachihara, K., Mizuno, A., et al. 1999, PASJ, 51, 895

Hartigan, P. 1993, AJ, 105, 1511

Hogerheijde, M. R., \& van der Tak, F. F. S. 2000, A\&A, 362, 697

Hogerheijde, M. R., van Dishoeck, E. F., Blake, G. A., \& van Langevelde, H. J. 1998, ApJ, 502, 315

Hollenbach, D. J., Yorke, H. W., \& Johnstone, D. 2000, Protostars and Planets IV, 401

Hughes, J., Hartigan, P., \& Clampitt, L. 1993, AJ, 105, 571

Hughes, J., Hartigan, P., Krautter, J., \& Kelemen, J. 1994, AJ, 108, 1071

Jørgensen, J. K., Schöier, F. L., \& van Dishoeck, E. F. 2002, A\&A, 389, 908

Kamp, I., van Zadelhoff, G.-J., van Dishoeck, E. F., \& Stark, R. 2003, A\&A, 397,1129

Kessler-Silacci, J., Augereau, J.-C., Dullemond, C. P., et al. 2006, ApJ, 639, 275 Knude, J., \& Høg, E. 1998, A\&A, 338, 897

Knude, J., \& Nielsen, A. S. 2001, A\&A, 373, 714

Koerner, D. W., \& Sargent, A. I. 1995, AJ, 109, 2138

Krautter, J., \& Reipurth, B. 1991, in ESO Scientific Reports (ESO press), 11, 127

Krautter, J., Alcala, J. M., Wichmann, R., Neuhauser, R., \& Schmitt, J. H. M. M. 1994, Rev. Mex. Astron. Astrofis., 29, 41

Krautter, J., Wichmann, R., Schmitt, J. H. M. M., et al. 1997, A\&AS, 123, 329

Lada, C. J. 1987, in 115: Star Forming Regions, ed. M. Peimbert, J. Jugaku, \& P. W. J. L. Brand (Dordrecht: Reidel), 1 IAU Symp.

Mannings, V., \& Sargent, A. I. 2000, ApJ, 529, 391

Marcy, G. W., \& Butler, R. P. 1995, BAAS, 27, 1379

Najita, J., Carr, J. S., \& Mathieu, R. D. 2003, ApJ, 589, 931 
Neuhäuser, R., Guenther, E. W., Wuchterl, G., et al. 2005, A\&A, 435, L13 Nürnberger, D., Chini, R., \& Zinnecker, H. 1997, A\&A, 324, 1036

Qi, C., Ho, P. T. P., Wilner, D. J., et al. 2004, ApJ, 616, L11

Rachford, B. L., Snow, T. P., Tumlinson, J., et al. 2002, ApJ, 577, 221

Rizzo, J. R., Morras, R., \& Arnal, E. M. 1998, MNRAS, 300, 497

Ruden, S. P. 1999, in The Origin of Stars and Planetary Systems, NATO ASIC Proc., 540, 643

Schwartz, R. D. 1977, ApJS, 35, 161

Simon, M., Ghez, A. M., \& Leinert, C. 1993, ApJ, 408, L33

Tachihara, K., Dobashi, K., Mizuno, A., Ogawa, H., \& Fukui, Y. 1996, PASJ, 48, 489
Tachihara, K., Toyoda, S., Onishi, T., et al. 2001, PASJ, 53, 1081

Teixeira, P. S., Lada, C. J., \& Alves, J. F. 2005, ApJ, 629, 276

Thi, W. F., van Dishoeck, E. F., Blake, G. A., et al. 2001, ApJ, 561, 1074

Thi, W.-F., van Zadelhoff, G.-J., \& van Dishoeck, E. F. 2004, A\&A, 425, 955

van Dishoeck, E. F., \& Black, J. H. 1988, ApJ, 334, 771

van Zadelhoff, G.-J., van Dishoeck, E. F., Thi, W.-F., \& Blake, G. A. 2001, A\&A, 377,566

van Zadelhoff, G.-J., Aikawa, Y., Hogerheijde, M. R., \& van Dishoeck, E. F. 2003, A\&A, 397, 789

Vilas-Boas, J. W. S., Myers, P. C., \& Fuller, G. A. 2000, ApJ, 532, 1038 Wilson, T. L., \& Rood, R. 1994, ARA\&A, 32, 191 
T. A. van Kempen et al.: Searching for gas-rich disks around T Tauri stars in Lupus, Online Material p 1

\section{Online Material}


T. A. van Kempen et al.: Searching for gas-rich disks around T Tauri stars in Lupus, Online Material p 2
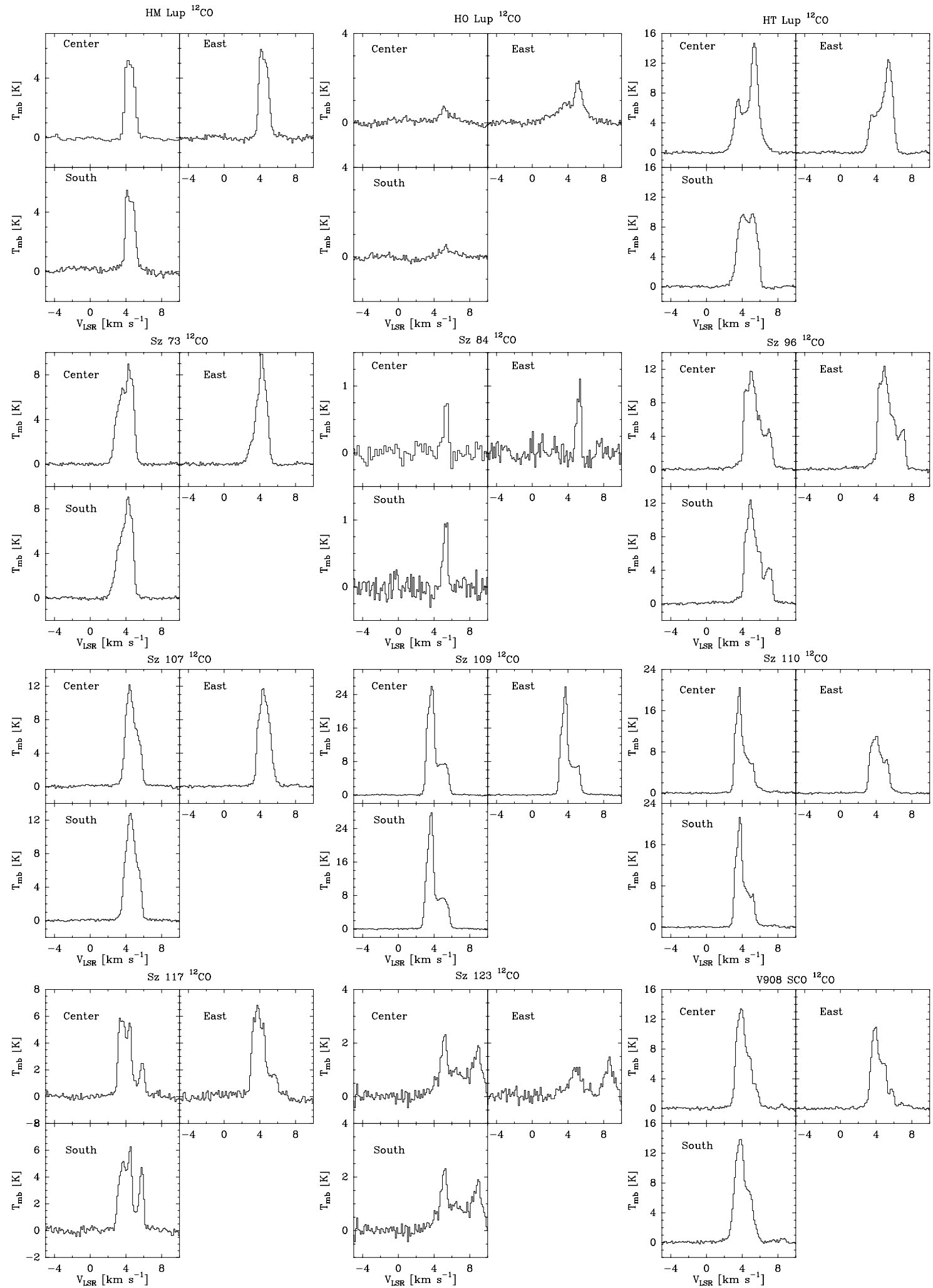

Fig. 6. ${ }^{12} \mathrm{CO} 3-2$ lines of selected sources in Lupus, with off-positions $30^{\prime \prime}$ south and east of source. 